NOTE

\title{
An apparatus for applying strong longitudinal magnetic fields to clinical photon and electron beams
}

\author{
Dale W Litzenberg ${ }^{1,4}$, Benedick A Fraass ${ }^{1}$, Daniel L McShan ${ }^{1}$, \\ Thomas W O'Donnell ${ }^{2}$, Donald A Roberts ${ }^{2}$, Fredrick D Becchetti ${ }^{2}$, \\ Alex F Bielajew ${ }^{3}$ and Jean M Moran ${ }^{1}$ \\ ${ }^{1}$ Department of Radiation Oncology, University of Michigan, Ann Arbor, MI 48109-0010, USA \\ 2 Department of Physics, University of Michigan, Ann Arbor, MI 48109-1120, USA \\ ${ }^{3}$ Department of Nuclear Engineering and Radiological Sciences, University of Michigan, \\ Ann Arbor, MI 48109-2104, USA \\ E-mail: litzen@umich.edu
}

Received 23 November 2000

\begin{abstract}
Monte Carlo studies have recently renewed interest in the use of the effect of strong transverse and longitudinal magnetic fields to manipulate the dose characteristics of clinical photon and electron beams. A $3.5 \mathrm{~T}$ superconducting solenoidal magnet was used to evaluate the effect of a longitudinal field on both photon and electron beams. This note describes the apparatus and demonstrates some of the effects on the beam trajectory and dose distributions for measurements in a homogeneous phantom. The effects were studied using film in air and in phantoms which fit in the magnet bore.

The magnetic field focused and collimated the electron beams. The converging, non-uniform field confined the beam and caused it to converge with increasing depth in the phantom. Due to the field's collecting and focusing effect, the beam flux density increased, leading to increased dose deposition near the magnetic axis, especially near the surface of the phantom. This study illustrates some benefits and challenges associated with the use of non-uniform longitudinal magnetic fields in conjunction with clinical electron and photon beams.
\end{abstract}

The dose delivered by electron and photon radiotherapy beams results from the ionization produced by the displacement and passage of electrons. These electrons are either primary particles, in the case of electron beams, or secondary particles set in motion by primary photon beams mostly through Compton scattering interactions. Electrons, once set in motion, scatter and lose energy due to elastic and inelastic collisions. This increases the penumbra width beyond that due to an extended source and collimator penetration, and can cause electronic

4 Address for correspondence: Department of Radiation Oncology, University of Michigan Health System, UH-B2C432C Box 0010, 1500 E Medical Center Drive, Ann Arbor, MI 48109-0010, USA. 
disequilibrium in the vicinity of heterogeneities. It has long been known that the application of a strong magnetic field to the scattering material, parallel to the beam axis, should reduce the lateral scattering of electrons (Bostick 1950). Monte Carlo simulations (Bielajew 1993) have demonstrated that very strong uniform longitudinal magnetic fields $(20 \mathrm{~T})$ could reduce the lateral spread of 10 and $20 \mathrm{MeV}$ electrons from many centimetres to a few millimetres. More moderate field strengths ( 3 to $6 \mathrm{~T}$ ) would also significantly reduce the penumbra. Bielajew's simulations have also shown that uniform longitudinal fields should not disturb local electron equilibrium. Additional Monte Carlo studies by Ramahi et al (2000) have calculated that longitudinal magnetic fields could potentially reduce lateral electron scatter in photon-beam radiotherapy for water-air-water heterogeneities. In that study, electronic equilibrium was improved in planar water-air-water slab geometries exposed to a $6 \mathrm{MV}$ photon beam by applying a longitudinal $0.5 \mathrm{~T}$ uniform field to the phantom.

The results of experiments using strong lateral magnetic fields to manipulate electron trajectories in phantoms have previously been reported (Shih 1975, Whitmire et al 1977, 1978, Paliwal et al 1978, 1979). In addition Monte Carlo simulations have been recently reported which used a model non-uniform transverse magnetic field with photon beams (Jette 2000, Li et al 2001).

In this study, an apparatus was designed and tested to investigate Monte Carlo predictions of the effect of longitudinal magnetic fields on megavoltage clinical photon and electron beams. This note describes the experimental set-up and shows the effects of 0.5 and $3 \mathrm{~T}$ magnetic fields on 10 and $20 \mathrm{MeV}$ electron beams and a $10 \mathrm{MV}$ photon. More detailed results and analysis will be presented in future publications.

The experiment was conducted using the high-energy (G50) gantry of a two-gantry racetrack microtron accelerator (MM50 Scanditronix, Uppsala, Sweden). The photon and electron beams of the high-energy gantry have been previously described (Karlsson et al 1992, 1993). The basic characteristics of the electron beams are as follows: electrons are accelerated in $5 \mathrm{MeV}$ increments with clinical beam energies ranging from 10 to $50 \mathrm{MeV}$. The collimator head is filled with helium to reduce scatter. The beam is magnetically scanned in a hexagonal pattern such that a uniform field results. A small scattering foil is used to increase the FWHM of the beams in clinical mode, for energies greater than $10 \mathrm{MeV}$. Measurements for these experiments were made with the clinical 10 and $20 \mathrm{MeV}$ electron beams with the scattering foil. Unmodified or 'elemental' electron beams may be extracted from the Microtron by removing the scattering foils and filters from the beam path. This reduces the FWHM of the $50 \mathrm{MeV}$ electron beam at isocentre from $5.0 \mathrm{~cm}$ to $1.5 \mathrm{~cm}$. Measurements were made with a $50 \mathrm{MeV}$ elemental pencil beam of electrons coincident with the central axis. The energy spread of this beam is $\pm 250 \mathrm{keV}$ at the gantry exit window.

Photon beams with energies of 10 and $25 \mathrm{MV}$ are also available on the G50 gantry. Uniform photon beams were produced by magnetically scanning the electron beam on a thin, high- $Z$ target in a concentric circular pattern such that a uniform field is produced. A purging magnet removes target-generated electrons from the field.

A superconducting solenoidal magnet (Intermagnetics General Corporation, Guilderland, New York), with a $20 \mathrm{~cm}$ diameter room-temperature bore, was used to produce a longitudinal field with a maximum strength of $3 \mathrm{~T}$ near the centre of the magnet. Simulation results (Bielajew 1993) confirm that a field strength of $3 \mathrm{~T}$ is sufficient observe a significant reduction in lateral electron scatter. A schematic of the experimental set-up is shown in figure 1. The properties of this magnet are shown in table 1 (Stern et al 1987). The superconducting magnet is wound from $\mathrm{Nb}-\mathrm{Ti}$ materials. The conductor consists of many continuous, fine, twisted filaments of $\mathrm{Nb}-\mathrm{Ti}$ embedded in a copper matrix. The magnet windings are layer wound and vacuum impregnated with epoxy on a cylindrical aluminium and epoxy-fibreglass form. The 


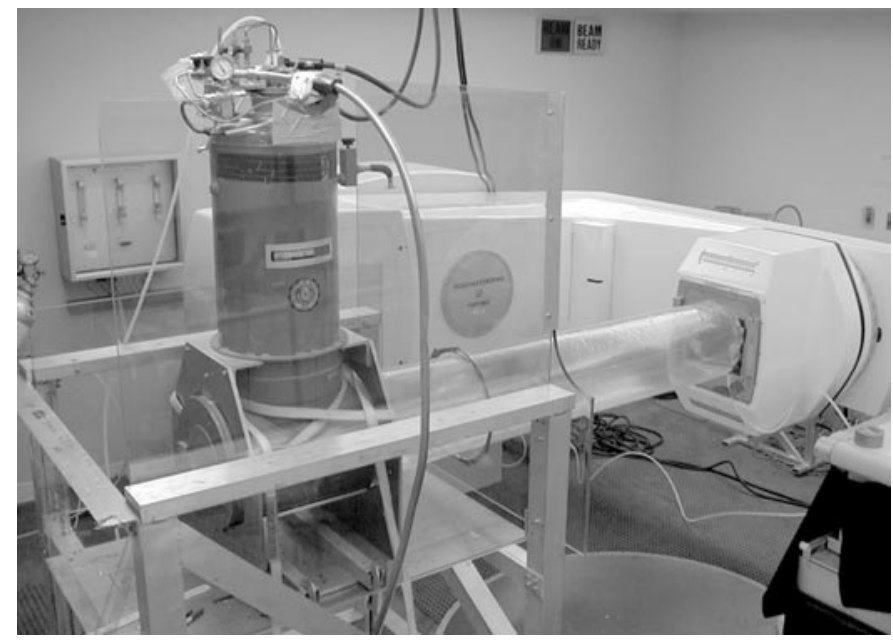

Figure 1. Photograph of the experimental set-up. The G50 gantry is shown at the right with the solenoidal magnet on the left. The beam was transported roughly $1.5 \mathrm{~m}$ from the gantry exit window to the front face of the cryostat through a thin-walled, helium-filled, plastic tube to minimize scatter. Cylindrical phantoms were placed in the bore of the magnet to make film measurements.

Table 1. Specifications of the superconducting solenoidal magnet.

\begin{tabular}{ll}
\hline Magnet coil length & $35.8 \mathrm{~cm}$ \\
Magnet coil inner diameter & $22 \mathrm{~cm}$ \\
Magnet coil outer diameter & $26 \mathrm{~cm}$ \\
Inductance & $\sim 12.5 \mathrm{H}$ \\
Stored energy (at $3.5 \mathrm{~T})$ & $\sim 100 \mathrm{~kJ}$ \\
Maximum central field & $3.5 \mathrm{~T}$ at $125 \mathrm{~A}$ \\
Power supply & $\mathrm{IGC}-180 \mathrm{M}( \pm 4 \mathrm{~V}, 180 \mathrm{~A})$ \\
Ramp time (0-3.5 T) & $25 \mathrm{~min}$ \\
Decay rate (persistent mode) & $\leqslant 10^{-4} \mathrm{~h}^{-1}$ \\
Cryostat length & $47 \mathrm{~cm}$ \\
Cryostat bore diameter & $20 \mathrm{~cm}$ \\
LHe Cryostat capacity & 101 \\
LHe to cool down & $50-701$ \\
LHe to refill & 151 \\
Power leads & Liquid He vapour cooled, non-retractable \\
Cryostat shielding & Vacuum inner shield, liquid nitrogen outer shield \\
Hold time (persistent mode) & $20 \mathrm{~h}$ \\
\hline
\end{tabular}

form is fitted to the air-bore wall of the cryostat and the cryostat is welded shut. The magnet is designed so that the magnetic axis and the cylindrical axis of the air-bore are coincident. The centre of the superconducting coil is $1.14 \mathrm{~cm}$ closer to one end of the cryostat than the other end by design.

The field map for this magnet was developed by a combination of measurements and calculations. The field strength on-axis was measured with a Hall probe. These data were then used to fit a calculation of the magnetic field to the observed data. The calculation was carried out by numerically integrating over the current distribution according to the law of Biot and Savart. The parameters of the calculation, including the length of the coil and its inner and outer radius, were adjusted so that the calculated values of the field strength on-axis agreed with the measured values to within $2 \%$, at the measured current. The measured and calculated 


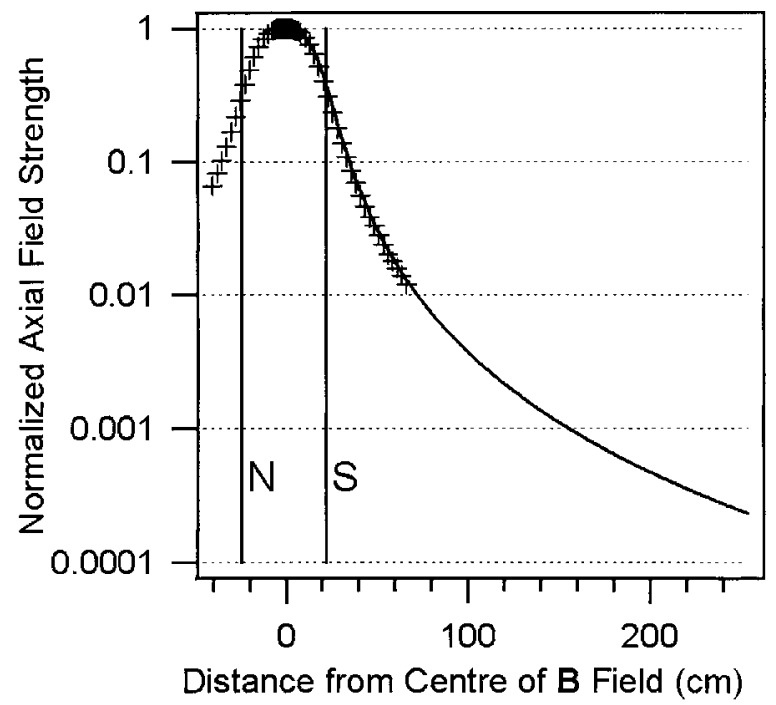

Figure 2. Measured (+) and calculated field strength along the magnetic axis, normalized to the maximum field strength. The lines labelled $\mathrm{N}$ and $\mathrm{S}$ show where the north and south ends of the cryostat are relative to the peak value of the magnetic field.

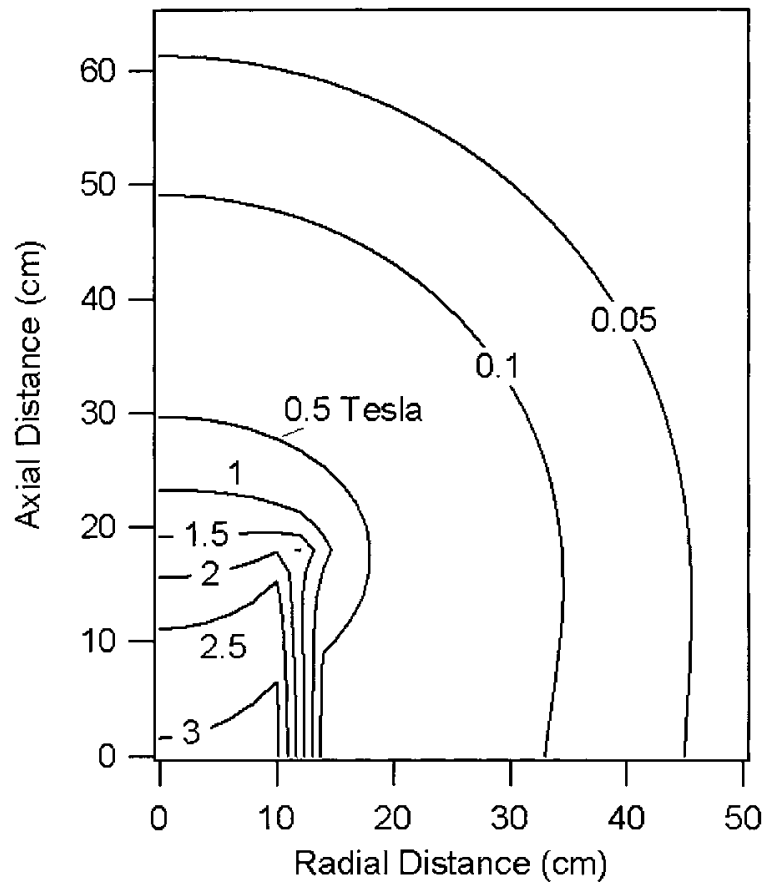

Figure 3. Lines of constant magnetic field strength for one quadrant of the superconducting solenoidal magnet.

on-axis field strength is shown in figure 2. The peak field strength along the axis is $22.4 \mathrm{~cm}$ from the end of the cryostat closest to the gantry. Figure 3 shows lines of constant field strength for one quadrant of the magnetic field obtained from these calculations. 
The magnet was placed as far as possible from the gantry to reduce effects of the magnetic field on the beam optics of the accelerator. The magnetic centre was $249 \pm 1 \mathrm{~cm}$ from the vacuum exit window. The calculated magnetic field strength, as described above, at the vacuum exit window is $7.5 \times 10^{-4} \mathrm{~T}$ for an unperturbed field. The primary component of the field is, in principal, parallel to the electron beam and only deflects those electrons that have a velocity component not directed along the magnetic axis. These particles with off-axis velocities arise from divergence and scattering of the beam.

The magnet was supported by, and fastened to, an aluminium frame, which was bolted to the treatment room floor. The experiment was designed such that the photon and electron beams would enter the magnet along the magnetic axis. The orientation and position of the magnet's cryostat, relative to the beam axis, was determined by iteratively acquiring radiographs of pencil-beam electrons on films registered to the front and back of the cryostat using XV film (Kodak, Ready Pack). This axis was parallel to the horizontal beam axis within $0.5^{\circ}$ and aligned within $1 \mathrm{~cm}$ at the front of the magnet.

To prevent degradation of the beam before it reached the magnet bore, a helium-filled plastic bag ( $29 \mathrm{~cm}$ diameter) was used to transport the beam from the treatment head to the front face of the magnet. The electrons were collimated using a $5 \mathrm{~cm}$ thick aluminium aperture with a $5 \mathrm{~cm}$ inner diameter opening. This collimator was placed in air, inside the magnet bore, with its front surface flush with the magnet cryostat's front face and the aperture centred on the axis of the magnet's air-bore.

Two cylindrical phantoms were designed and built to fit inside the magnet's $20 \mathrm{~cm}$ diameter bore. One phantom consisted of cylindrical polystyrene plates, $19 \mathrm{~cm}$ in diameter and of varying thickness, to measure dose distributions perpendicular to the beam at the desired depth. The plates were held together by three Lexan dowels uniformly spaced around the inside perimeter of the cylinder. The ends of the dowels were threaded to allow the plates to be compressed together. Typical polystyrene phantoms ranged from 17 to $40 \mathrm{~cm}$ thick depending on the beam energy and modality. A second phantom was constructed from a machinable cylinder of urethane resin, which has a density of $1.04 \mathrm{~g} \mathrm{~cm}^{-3}$. The material was machined into a $19 \mathrm{~cm}$ diameter cylinder, $30 \mathrm{~cm}$ long and cut in half along its cylindrical axis. Film was then placed between the two halves in order to make depth-dose measurements. Film (Kodak, XV Ready Pack) was cut to fit the in the phantom in a darkroom and sealed with light-tight tape. For depth dose measurements the film was placed so that the film was flush against the hand-taped edge of the pack.

The radiation field along the $2.5 \mathrm{~m}$ flight-path to the magnet, with and without the magnetic field present, was observed by placing films perpendicular to a uniform $10 \mathrm{MeV}$ electron beam. The first film was taped vertically onto the gantry head exit window in air with the helium bag pressing against the back side of the film. The second film was placed in air, against the distal end of the helium bag on the front face of the magnet's cryostat. The third film was placed in air on the back face of the magnet's cryostat. These distances corresponded to $70 \mathrm{~cm}, 226 \mathrm{~cm}$ and $273 \mathrm{~cm}$ respectively from the source. Figure 4 shows the uniformity of the radiation field exiting the treatment head when no magnetic field was present. Figure 5 shows the digitized films for the $10 \mathrm{MeV}$ beam when a magnetic field with a peak strength of $3 \mathrm{~T}$ was present. It can be seen in figure 5(a) that the beam exiting the collimator is no longer uniform over the field. This beam approaches the magnet on-axis, through the magnet's fringe field, in a helium atmosphere. This non-uniform beam has collapsed to a circle with significant radial structure at the front face of the magnet where the axial field strength is approximately $0.9 \mathrm{~T}$ (figure $5(b)$ ). The beam then enters air and passes through a $5 \mathrm{~cm}$ diameter, $5 \mathrm{~cm}$ thick aluminium collimator before traversing the bore of the magnet. The collimator has removed the outer portion of the beam and the remaining portion has been focused to a FWHM of $1.54 \mathrm{~cm}$ as it leaves the solenoid (figure $5(c)$ ). 


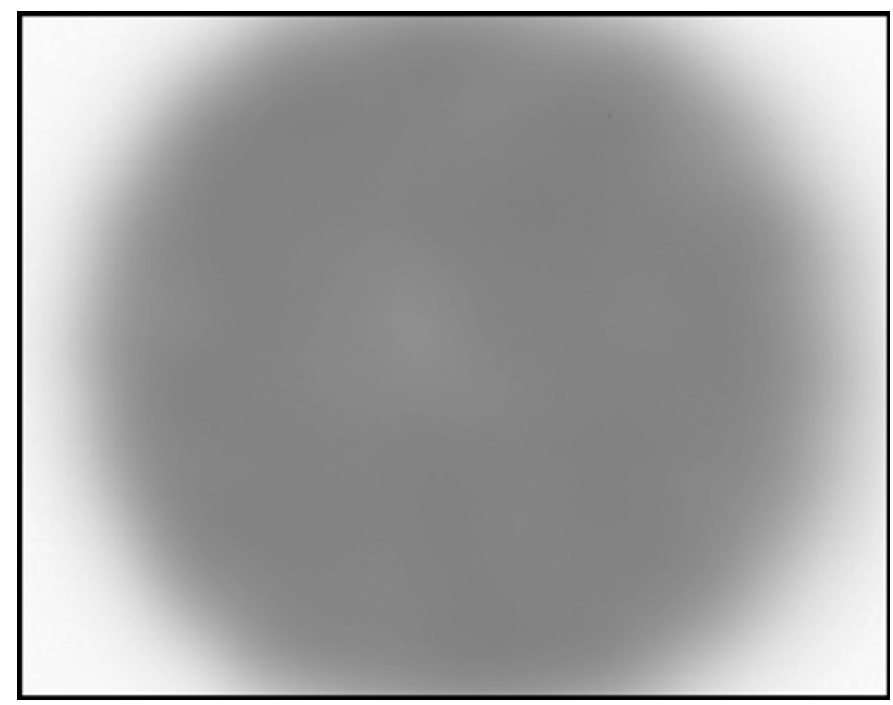

Figure 4. Digitized film showing a uniform $10 \mathrm{MeV}$ electron beam exiting the gantry head when no magnetic field is present in the solenoid.

A comparison of figures 4 and 5(a) shows that the magnetic field has interfered with the beam transport optics. This indicates that the steel in the gantry has acted as a partial magnetic yoke, warping the solenoidal field to some degree. Instead of producing a uniform field as expected from the uniform scan pattern, most of the dose is in a semicircular pattern, with the chord vertical. As the beam approaches the magnet each electron is subject to the force given by

$$
\boldsymbol{F}=q \boldsymbol{v} \times \boldsymbol{B}
$$

where $q$ is the charge on the electron, $\boldsymbol{B}$ is the magnetic field and $\boldsymbol{v}$ is the velocity of the electrons, which is only slightly varying in the helium atmosphere. The converging fringe field collects and focuses the beam into the magnet. Electrons whose velocities are directed mainly along the magnetic axis experience relatively little force and are focused into the magnet along the converging magnetic field lines forming the dark central peak. Electrons scattered through larger angles that would normally be removed by electron cone collimators are also focused into the magnet and form the continuum of dose outside the central peak. The backscatter from the edge of the $5 \mathrm{~cm}$ inner diameter collimator is also seen. The beam is then collimated to $5 \mathrm{~cm}$ diameter as it enters the cryostat, leaving primarily the central peak, as seen at the exit of the cryostat on the films. The beam has travelled through $47 \mathrm{~cm}$ of air in the bore of the magnet at this position, which has increased the FWHM diameter of the central peak due to scattering. This focusing or pinching effect on the beam trajectory, as it approaches the magnet, before reaching the phantom, has been previously demonstrated by Monte Carlo simulation (Weinhous et al 1985).

The effect of the $3 \mathrm{~T}$ magnetic field on the beam trajectory and the dose profiles was significant for all electron beam energies. An example distribution, measured with film, is shown in figure 6 for $20 \mathrm{MeV}$ electrons with $0 \mathrm{~T}$ and $3 \mathrm{~T}$ magnetic fields. Note that the greyscale windowing and levelling on figures $6(a)$ and $6(b)$ are not the same; this is to highlight the features on each film. Each image is scaled to $100 \%$ at $3 \mathrm{~cm}$ on the central axis. The 10, 20, 50,80 and $100 \%$ isodose lines are shown. The distribution shown in figure $6(a)$ is as expected for $20 \mathrm{MeV}$ electrons. In this case, no magnetic field was present and the beam was collimated 


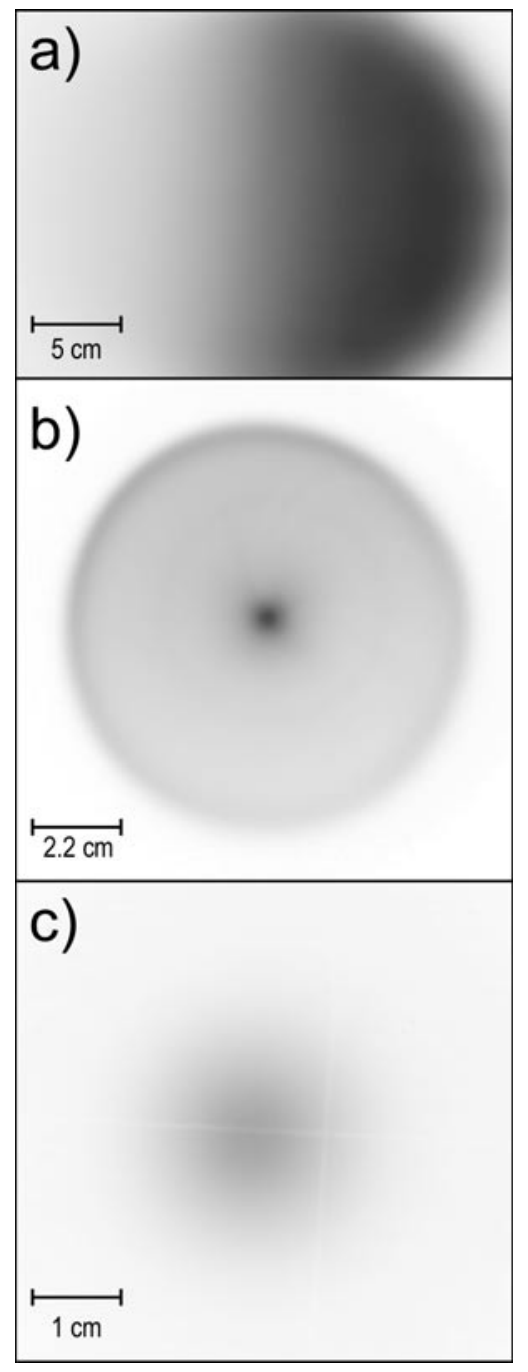

Figure 5. Digitized film showing a lateral profile of a $10 \mathrm{MeV}$ electron beam when the peak magnetic field in the solenoid is $3 \mathrm{~T}$. Part (a) Shows that the beam exiting the gantry head is not uniform, (b) shows the beam at the front surface of the magnet's cryostat and (c) shows the beam at the back surface of the cryostat.

to $5 \mathrm{~cm}$ in diameter. Figure $6(b)$ shows the distribution produced by the beam when a $3 \mathrm{~T}$ field is present under otherwise identical conditions. Notice that the field has perturbed the distribution in a number of ways. Firstly, the width of the beam at the surface of the phantom is smaller $(3.3 \mathrm{~cm}$ FWHM compared with $4.9 \mathrm{~cm})$. Secondly, the distribution is pinched inwards at a depth $(1.8 \mathrm{~cm}$ FWHM compared with $5 \mathrm{~cm}$ at a depth of $3 \mathrm{~cm})$. The typical bowing out of the distribution toward the end of the electron range (figure $6(a)$ ) is not seen with the magnetic field present (figure $6(b)$ ). This effect was also seen for the other electron beam energies.

Films exposed with the magnetic field present had a much higher maximum optical density (OD). A 40 MU exposure, with no magnetic field, gave an OD of 0.33 at a depth of $3 \mathrm{~cm}$. In the presence of a $3 \mathrm{~T}$ magnetic field, the OD was 1.6 at the same depth. This is the result of the converging, non-uniform magnetic field collecting and focusing electrons onto the magnetic axis. Thus many of the electrons that normally would have been scattered out of the beam or removed by an applicator in the absence of a magnetic field now enter the phantom. In addition, the converging magnetic field has reduced the effective aperture causing the electron 


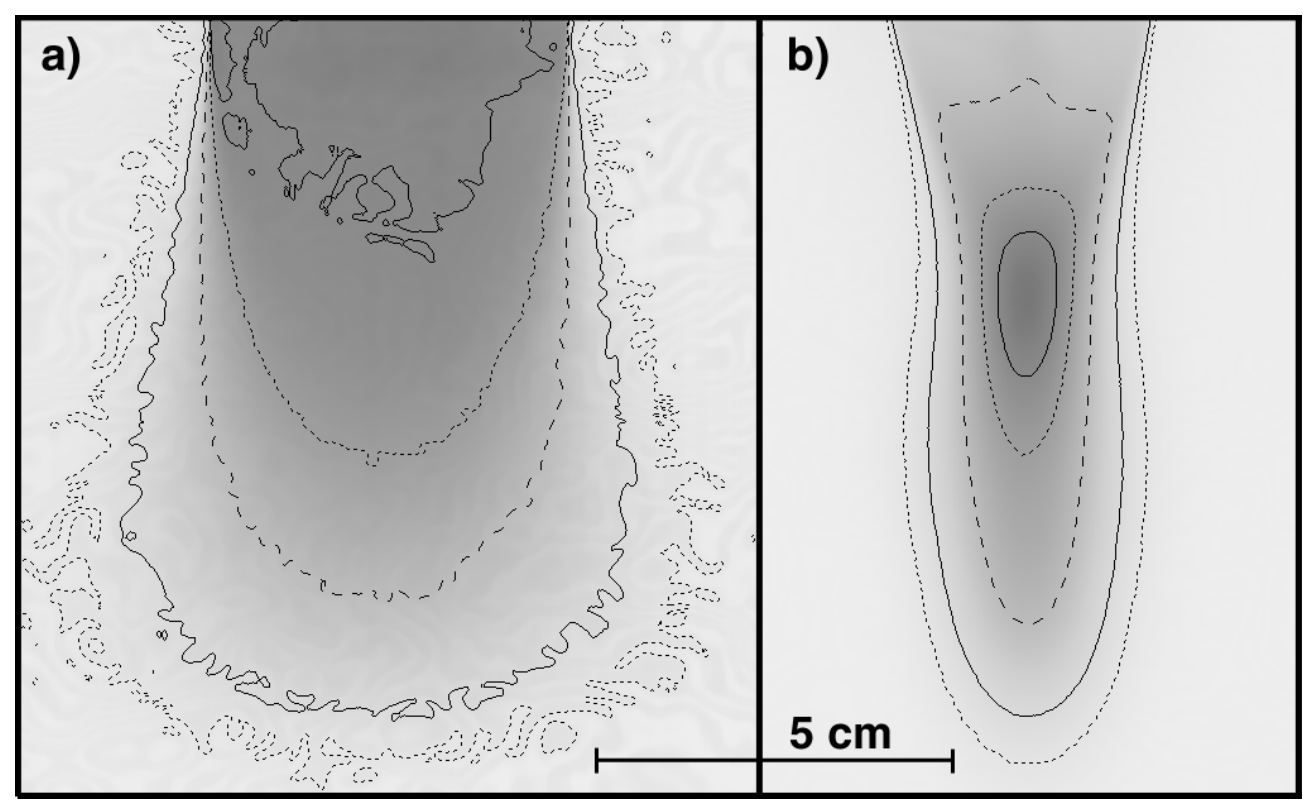

Figure 6. Digitized film showing the depth dose characteristics of a $20 \mathrm{MeV}$ electron beam after passing through the $5 \mathrm{~cm}$ collimator in the magnet's bore. Part $(a)$ shows the beam when no magnetic field is present, $(b)$ shows the beam when the peak magnetic field in the solenoid is $3 \mathrm{~T}$. Both images are at the same scale. The images are independently scaled to $100 \%$. The $10,20,50$, 80 and $100 \%$ isodose lines are shown.

flux density to greatly increase. This effect indicates that dosimetry measurements conducted with no magnetic field would not be valid in the presence of a strong solenoidal field as previously believed from simulation results which approximated the field as uniform in the phantom (Bielajew 1993). However, an aperture placed between the exit window of the gantry and the front of the magnet could be used to reduce the angular acceptance of the magnet, reducing the observed collecting and focusing effects.

There is also a rather large field-strength gradient over the length of the distribution shown in figure $6(b)$. The front of the phantom is located about $17 \mathrm{~cm}$ from the magnetic centre, where the field strength is approximately $1.7 \mathrm{~T}$ (figure 2 ). The field strength $10 \mathrm{~cm}$ into the phantom, or $7 \mathrm{~cm}$ from the magnetic centre, is approximately $2.8 \mathrm{~T}$. Therefore, as the electrons lose energy and scatter through larger angles they experience a much stronger force that confines and focuses the beam. While it is possible to modify the field gradients near the magnet with trim coils to tailor this focusing effect, the beam would still have to approach the magnet on axis through a dipole field and therefore experience a converging field with a strong gradient. It is possible that this gradient could be located outside the patient, though this would require a more sophisticated and expensive magnet system.

The relevant Monte Carlo studies in the literature simulate physical situations that are slightly different from those present in this experiment. The simulations presented by Weinhous use an extended field to account for electron beam transport phenomena before reaching the phantom. Simulations by Bielajew (1993) use a uniform magnetic field inside the phantom and no field outside the phantom. The beam trajectory results found by Weinhous, as the beam approaches the phantom, were observed in these experiments but the predicted Bragg peak effect is not observed, as suggested by Bielajew. Simulations by Bielajew show that in 


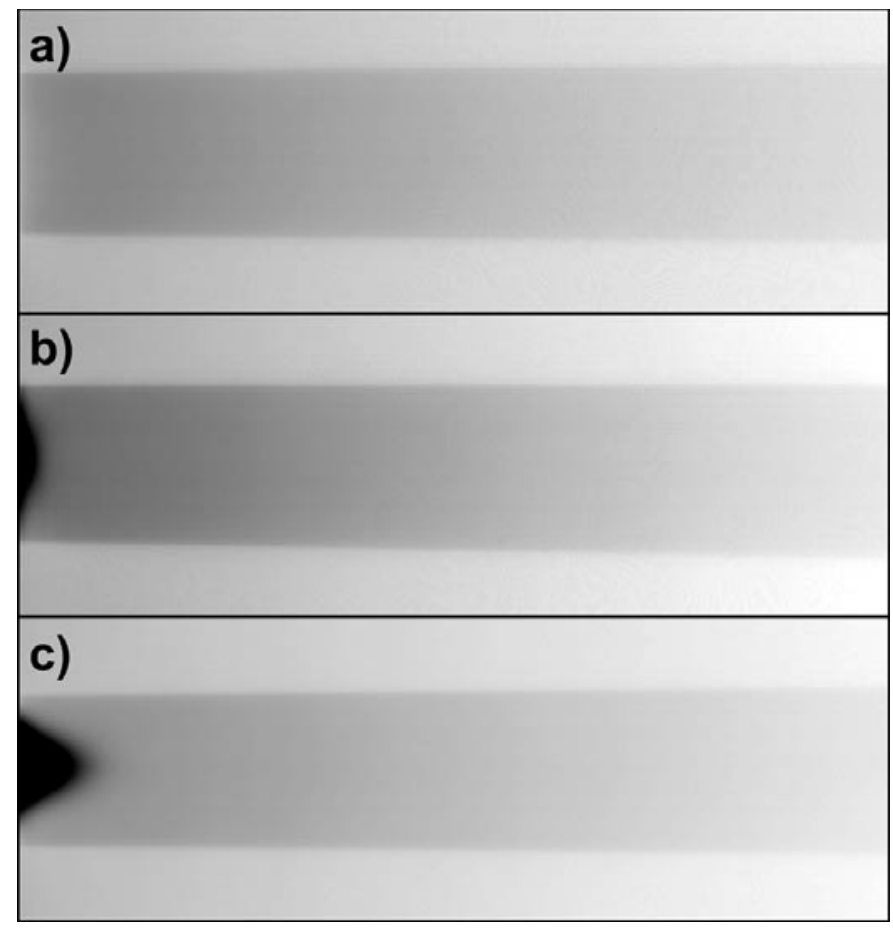

Figure 7. Digitized film showing the depth dose characteristics of a $10 \mathrm{MV}$ photon beam after passing through the $5 \mathrm{~cm} \mathrm{Al} \mathrm{collimator} \mathrm{in} \mathrm{the} \mathrm{magnet's} \mathrm{bore.} \mathrm{Part} \mathrm{(a)} \mathrm{Shows} \mathrm{the} \mathrm{beam} \mathrm{when} \mathrm{no}$ magnetic field is present, $(b)$ shows the beam when the peak magnetic field in the solenoid is $0.5 \mathrm{~T}$ and $(c)$ shows the beam when the peak magnetic field in the solenoid is $3.0 \mathrm{~T}$.

a uniform longitudinal magnetic field the beam penumbra should be reduced and predict a fluence enhancement at greater depths in the phantom, due to the lateral confinement of the electron trajectories in the phantom. In the set-up presented here the magnetic field is nonuniform and converging over the entire trajectory of the electron beam. This leads to slightly different results than predicted in the simulations, in that the beam converges as it travels into the phantom. This results in a loss of charged-particle equilibrium making dosimetry more difficult since dose is no longer equal to the collisional kerma. In addition, the surface contamination effects due to collecting and focusing low-energy scattered electrons were not predicted.

Using the same set-up described above for electron beams, the effect of a longitudinal magnetic field on the characteristics of a $10 \mathrm{MV}$ photon beam in a homogeneous phantom were also observed. This set-up was not optimized for photon experiments in the beam was collimated with the same $5 \mathrm{~cm}$ thick aluminium collimator used for the electrons. Time constraints, the long magnetic field ramp-time and safety considerations with removing and inserting the collimator in the presence of a strong magnetic field prevented the use of a more appropriate collimator. Due to the relatively low attenuating characteristics of aluminium compared with lead, tungsten or cerrobend, the dose behind the collimator was approximately $20 \%$ lower than the primary beam.

The films in figure 7 show the depth dose characteristics of the 10 MV beam. Figure 7(a) shows the beam when no magnetic field is present. When a magnetic field is applied, contaminant electrons are collected and focused near the surface of the phantom. The focusing 
effect is larger for the $3 \mathrm{~T}$ beam (figure $7(c)$ ) than for the $0.5 \mathrm{~T}$ beam (figure $7(b)$ ). These electrons probably originate from scattering interactions in the multileaf collimator $(10 \mathrm{~cm} \times 10 \mathrm{~cm}$ at isocentre), the plastic bag filled with $\mathrm{He}$ and the aluminium collimator. As noted earlier, a purging magnet in the G50 gantry removes contaminant electrons generated in the tungsten target. Beam profiles were extracted at $10 \mathrm{~cm}$ depth from the films shown in figure 7. No significant differences were seen in the field and shoulder widths for the homogeneous phantom. (Reductions in the penumbra are primarily expected in low-density heterogeneities where electrons have longer ranges.)

While the apparatus presented here for preliminary studies is not optimized for clinical use, much can be learned from these results about improving the design parameters. For example, figure 5(a) illustrates that the magnet and beam delivery optics in the gantry should to be considered simultaneously in the design process. Simulations have not been carried out that include warping of the solenoidal field due to a ferrous gantry. In a system intended for clinical use the magnet would be much closer to the gantry, producing a much more pronounced effect. Figure 5(b) confirms that the field size is strongly affected by the converging solenoidal field. This focusing or pinching effect on the beam trajectory, as it approaches the magnet, before reaching the phantom, has been previously demonstrated by Monte Carlo simulation (Weinhous et al 1985). The entire gantry and magnet system must be designed to accommodate strong fields and prevent fringe fields from interfering with the optimal delivery of radiation. Such a design may incorporate a $\mathrm{C}$-arm gantry that would serve as a magnetic yoke producing a more uniform field across a large gap centred about the isocentre. This could potentially produce a more uniform magnetic field, leading to a more uniform radiation field. The collecting and focusing effects of the solenoidal field on scattered electrons has been shown in these results. Any system using a strong longitudinal magnetic field should be designed to minimize the scattering of the primary beam in the gantry head and in air. For this reason, the beam should be transported in vacuum or helium as long as possible.

In summary, we describe an experimental set-up, using a superconducting solenoidal magnet, to investigate the effect of strong longitudinal magnetic fields on clinical electron and photon beams. The effect of the magnetic field on the beam trajectory was larger than anticipated and displayed a magnetic focusing and collimating effect on the beam. The phantoms were positioned near one end of the magnetic bore where the electrons experienced a strong field-strength gradient over their stopping range. In addition, the converging, nonuniform field not only confined the beam but also caused it to converge with increasing depth into the phantom. Due to the collecting and focusing effect of the solenoidal field the beam flux density increased, leading to increases in the observed dose deposition along the magnetic axis, especially near the surface of the phantom. Although the technique introduces some difficulties with focusing scattered electrons into the field, it shows promise for confining and focusing primary and secondary electrons during treatment.

\section{Acknowledgments}

This experiment required a significant amount of logistical collaboration and cooperation among several groups. We would like to thank Theodore Lawrence and Kathy Lash of the Department of Radiation Oncology for graciously allowing us to conduct this experiment in the clinic and the personnel at UM Medical Center OSEH Department for assistance with many safety issues. Indrin Chetty provided Monte Carlo simulation support to help minimize scattering during beam transport. Robin Kelly provided valuable safety services and assisted in conducting the experiment with Lianyan Liu assisting as well. 


\section{References}

Bielajew A F 1993 The effect of strong longitudinal magnetic fields on dose deposition from electron and photon beams Med. Phys. 20 1171-9

Bostick W H 1950 Possible techniques in direct-electron beam tumor therapy Phys. Rev. 77 564-5

Jette D 2000 Magnetic fields with photon beams: Monte Carlo calculations for a model magnetic field Med. Phys. 27 2726-38

Karlsson M, Nyström H and Svensson H 1992 Electron beam characteristics of the 50-MeV racetrack microtron Med. Phys. 19 307-15

1993 Photon beam characteristics on the MM50 racetrack microtron and a new approach for beam quality determination Med. Phys. 20 143-9

Li X A, Reiffel L, Chu J and Naqvi S 2001 Conformal photon-beam therapy with transverse magnetic fields: a Monte Carlo study Med. Phys. 28 127-33

Paliwal B R, Thomadsen B R and Wiley A L 1979 Magnetic modification of electron beam dose distributions Acta Radiol. Oncol. 18 57-64

Paliwal B R, Wiley A L, Wessels B W and Choi M C 1978 Magnetic field modification of electron-beam dose distributions in inhomogenous media Med. Phys. 5 404-8

Ramahi S W, Naqvi S A and Chu J 2000 Achieving a smaller penumbra region for better planning in conformal radiotherapy by using a longitudinal magnetic field CD-ROM Proc. World Congress on Medical Physics and Biomedical Engineering (23-28 July 2000)

Shih C C 1975 High energy electron radiotherapy in a magnetic field Med. Phys. 2 9-13

Stern R L et al 1987 Tests of a large air-core superconducting solenoid as a nuclear-reaction-product spectrometer Rev. Sci. Instrum. 58 1682-93

Weinhous M S, Nath R and Schultz R J 1985 Enhancement of electron beam dose distributions by longitudinal magnetic fields: Monte Carlo simulations and magnet system optimization Med. Phys. 15 598-603

Whitmire D P, Bernard D L and Peterson M D 1978 Magnetic modification of the electron-dose distribution in tissue and lung phantoms Med. Phys. 5 409-17

Whitmire D P, Bernard D L, Peterson M D and Purdy J A 1977 Magnetic enhancement of electron dose distribution in a phantom Med. Phys. 4 127-31 